\title{
Deformidades e incapacidades dos hemofílicos do Centro de Hemoterapia e Hematologia do Espírito Santo, Brasil
}

\section{Deformities and disabilities of hemophilics undergoing treatment at the Hemoterapy and Hematology Centre of Espirito Santo, Brazil}

\author{
Elem Guimarães dos Santos ${ }^{1}$, Linger Laci Portes², Anne Guimarães \\ Santana $^{3}$, Edson Theodoro dos Santos Neto ${ }^{4}$
}

SANTOS, E. G.; PORTES, L. L.; SANTANA, A. G.; SANTOS NETO, E. T. Deformidades e incapacidades dos hemofílicos do Centro de Hemoterapia e Hematologia do Espírito Santo, Brasil. Rev. Ter. Ocup. Univ. São Paulo, v. 18, n. 2, p. 86-94, maio/ago., 2007.

\begin{abstract}
RESUMO: A hemofilia é um grave distúrbio hereditário da coagulação sanguínea que pode resultar em diversas deformidades e incapacidades no corpo, permanentes ou transitórias, além das consequiências psicossociais nos indivíduos afetados. A proposição do presente estudo é identificar quais as principais seqüelas e incapacidades que acometem os portadores de hemofilia do Centro de Hemoterapia e Hematologia do Espírito Santo (HEMOES). Além de discutir a importância da inserção do serviço de terapia ocupacional como parte integrante do atendimento integral à saúde desses pacientes. Foram analisados 161 prontuários de pacientes atendidos no HEMOES de 2001 a 2004 anotando-se os acometimentos à hemofilia por um roteiro de pesquisa estruturado. Os 161 prontuários corresponderam a cerca de $40 \%$ do total de hemofílicos cadastrados no estado. A maioria era do sexo masculino $98 \%$ e o tipo de coagulopatia mais comum foi hemofilia A (77\%). Quanto aos níveis de severidade, predominaram dos tipos de deficiência grave e moderada que somados totalizaram $43 \%$ dos casos, enquanto o tipo leve representou $16 \%$ dos casos. As incapacidades e deformidades em regiões articulares do corpo corresponderam a $89 \%$ de acometimentos, enquanto as regiões musculares $11 \%$. Conclui-se que o perfil dos pacientes hemofílicos atendidos no HEMOES destacase pelo alto percentual de seqüelas e incapacidades, gerando a necessidade de atuação profissional desde o nível biológico até o nível psicossocial. Isso ressalta a importância da implantação, junto à equipe multiprofissional, do serviço de Terapia Ocupacional para atendimento integral à saúde.
\end{abstract}

DESCRITORES: Hemofilia A/complicação. Serviço de Hemoterapia. Terapia ocupacional.

\footnotetext{
${ }^{1}$ Terapeuta Ocupacional da Prefeitura Municipal de Marechal Floriano - Secretaria de Ação Social, Centro de Referência da Assistência Social. Graduação em Terapia Ocupacional e Pós-graduação em Reabilitação de Membro Superior pela FAESA e-mail: elemsantos@hotmail.com

${ }^{2}$ Técnico em Enfermagem do Centro de Hemoterapia e Hematologia do Espírito Santo. Graduação em Terapia Ocupacional e Pósgraduação em Reabilitação de Membro Superior pela FAESA e-mail: terportes@yahoo.com.br.

Consultório particular em Serviços de Terapia Ocupacional - Vitória- ES. Graduação em Terapia Ocupacional pela UFMG e PósGraduação em Terapia da Mão pela Associação de Terapeutas Ocupacionais do Estado do Rio de Janeiro.

${ }^{4}$ Graduação em Odontologia pela UFES e mestrando em Saúde Coletiva pelo Programa de Pós-graduação em Atenção à Saúde Coletiva da Universidade Federal do Espírito Santo - UFES e-mail: edson.neto@bol.com.br.
}

Endereço para correspondência: Edson Theodoro dos Santos Neto. Rua Guilherme Bassini, 286. São Pedro I. Vitória, ES. CEP: $29030-720$. 


\section{INTRODUÇÃO}

A hemofilia é classificada pela hematologia como uma coagulopatia ou distúrbio hereditário da coagulação sanguínea causada pela deficiência dos fatores VIII e IX da coagulação (proteínas plasmáticas do sangue responsáveis pela ativação do processo de coagulação sanguínea). Existem dois tipos de hemofilia, a do tipo A que indica a deficiência do fator VIII e a hemofilia do tipo B, caracterizada pela deficiência do fator IX. Estas hemofilias não são distinguidas clinicamente, pois ambas se manifestam sob as formas leve, moderada, severa ou grave. Segundo Lorenzi et al. (2003), os genes hemofílicos (A e B) resultam de uma mutação que se localiza no cromossomo $X\left(X^{\mathrm{h}}\right)$, atuando como genes recessivos. Por isso, é uma doença hereditária recessiva ligada ao sexo que raramente se manifesta no sexo feminino.

$\mathrm{Na}$ hemofilia, o processo de coagulação sanguínea, que é um integrante básico do sistema de hemostasia, é alterado caracterizando-se por repetidos sangramentos espontâneos causados por traumas mínimos que envolvem quaisquer órgãos. A deficiência dos fatores de coagulação do sangue causa modificação nesse sistema levando a um quadro hemorrágico, tendo maior prevalência de sangramentos no sistema osteoarticular (WILLIAM et al. 1990).

Quanto aos aspectos clínicos, a hemofilia se manifesta também através de hemorragias que podem ocorrer no sistema músculo-esquelético, gastrintestinal, genitourinário, sistema nervoso central, mucosas e pele, sendo a maior prevalência de acometimento do sistema músculoesquelético. Nesse caso, a hemofilia se manifesta principalmente através das hemartroses (sangramentos intra-articulares) sendo a manifestação mais comum e debilitante da hemofilia, que em passado mais recente levava a invalidez permanente um grande número de pacientes pelas lesões osteoarticulares irreversíveis (WINTROBE, 1998).

A hemofilia grave e moderada são as que mais causam deformidades e limitações às condições de vida do doente, levando, por vezes, a incapacidades permanentes e sequielas irreversíveis que influenciam no modo de vida da pessoa. Para Caio et al. (2001), a hemofilia grave é a mais dolorosa, incapacitante e deformante e segundo Lee et al. (2000) leva a limitações na vida do paciente, tanto a nível orgânico como pessoal. Por suas diversas limitações, os portadores de hemofilia necessitam de cuidados especiais como transfusões de sangue e fatores de coagulação, sem os seria impossível sua sobrevivência.

Depois de ser negligenciado por muitos anos, o governo federal brasileiro criou em 1980 o Pró-Sangue, um programa especial voltado para área de hemoderivados (BRASIL, 1980). O objetivo maior do programa foi a implantação dos hemocentros, difundindo assim conceitos sobre a doação de sangue voluntária e gratuita, sobre o sangue como um bem não mercantil, sobre os programas de captação de doadores de sangue, sobre a obrigatoriedade dos exames sorológicos e o fracionamento adequado do sangue (BRASIL, 2003). O Pró-Sangue representou a primeira ação direta e coordenada para o setor de hemoterapia por parte do governo implantando hemocentros nas capitais estaduais, considerados unidades de referência que atuariam no controle, processsamento e distribuição do sangue para uma determinada região, além de funcionar como centro de treinamento de pessoal, pesquisa e tratamento das doenças ligadas ao sangue.

Entretanto, esse tema ganhou mais notoriedade a partir de 1985 em decorrência do aparecimento de casos de hepatite e AIDS (Síndrome da Imunodeficiência Adquirida) transmitidas por transfusões sanguíneas, levando o Ministério da Saúde a estabelecer medidas rigorosas no sentido de oferecer maior segurança aos doadores e receptores de sangue e hemoderivados (BRASIL, 2003, 2004). Com a criação do Pró-Sangue e a concomitante proibição da doação remunerada, a melhoria da qualidade da hemoterapia brasileira ficou evidentemente reconhecida nacionalmente e internacionalmente.

Atualmente, conforme a Word Federation of Haemophilia (2002), há cerca de 35 (trinta e cinco) hemocentros distribuídos em todo o país nas regiões norte, nordeste, sul, sudeste e centro-oeste. Por isso, no Brasil os hemocentros assumem papel fundamental, pois é uma "unidade de referência que controla, processa, distribui o sangue para pesquisas, tratamentos de doenças ligadas ao sangue e é o órgão centralizador das estatísticas" (BONFIM; CAMPOS, 2001, p. 69).

No caso específico do Estado do Espírito Santo, a história da implantação do Centro de Hemoterapia e Hematologia do Espírito Santo (HEMOES) esteve diretamente relacionada à história da hemoterapia no Brasil. Isto por que apresentava uma situação problemática: a maioria dos bancos de sangue pertencia à rede privada retardando a implantação do projeto durante 13 anos (BONFIM; CAMPOS, 2001)

Depois de toda a estruturação histórica, atualmente os hemocentros se consolidam como o principal centro de referência para atendimento dos portadores da hemofilia, quando estes apresentam quadros hemorrágicos ou quando necessitam de atendimento especializado reunindo profissionais de diversas áreas. Diante disso, a Terapia 
Ocupacional funciona como uma profissão essencial, inserida numa equipe multidisciplinar para tratamento dos portadores de hemofilia que visa desenvolver autonomia e independência no desempenho de suas atividades de vida diária, de vida prática, de vida produtiva e de lazer, através da reabilitação física, mental e social (DE CARLO; LUZO, 2004).

Diante das bases apresentadas o presente estudo se propõe identificar quais as principais seqüelas e incapacidades que acometem os portadores de hemofilia do Centro de Hemoterapia e Hematologia do Espírito Santo. Além de discutir a importância da inserção do serviço de terapia ocupacional como parte integrante do atendimento integral à saúde desses pacientes.

\section{METODOLOGIA}

Trata-se de um estudo descritivo quantitativo realizado no HEMOES através do levantamento de dados de prontuários dos pacientes portadores de hemofilia do setor de transfusão e farmácia.

O HEMOES foi inaugurado, no dia $1^{\circ}$ de outubro de 1999 localiza-se na cidade de Vitória, capital do estado do Espírito Santo, sendo construído através de uma parceria entre o governo federal e estadual. É um banco de sangue público, sem fins lucrativos, que tem por objetivo "fornecer sangue, hemocomponentes e hemoderivados, com qualidade e quantidade necessária à demanda dos hospitais do estado" (BONFIM; CAMPOS, 2001, p.69) e como missão "executar as políticas estaduais relativas à hematologia e hemoterapia em consonância com a política do Ministério da Saúde, visando à prestação de serviços de qualidade" (HEMOES, 2001, p. 4).

$\mathrm{Na}$ área da hematologia, é o centro de referência no atendimento aos pacientes hemofílicos desde o ano de 2001. Dentro de suas finalidades de prestar assistência hematológica e hemoterápica à população, o HEMOES é o serviço encarregado de centralizar a assistência transfusional aos hemofílicos da rede pública e privada. A maioria dos casos atendidos são intercorrências diárias devido a sangramentos espontâneos ou causados por algum trauma. No HEMOES o serviço de atendimento aos hemofílicos reúne profissionais da área da enfermagem, hematologia, ortopedia, serviço social e fisioterapia. Conforme dados do setor de farmácia e transfusão do HEMOES, em todo o estado estão cadastrados cerca de 400 (quatrocentos) hemofílicos, destes, aproximadamente 170 (cento e setenta) se dirigem ao HEMOES para atendimento ambulatorial e prescrição dos fatores de coagulação.
A Amostra foi constituída por 161(cento e sessenta e um) prontuários de pacientes portadores de hemofilia assistidos pelo HEMOES no período de 2001 a 2004. Para isso, utilizou-se um instrumento de coleta de dados composto por um roteiro de pesquisa com questões fechadas avaliando-se às seguintes variáveis: sexo, tipo de hemofilia, o tipo de deficiência de fator coagulante e as áreas de acometimento das deformidades, coletadas no período de 01 a 31 de julho de 2004.

A classificação das hemofilias A e B baseou-se na atividade residual dos fatores VIII e IX, considerando o nível normal de fator médio igual a $100 \mathrm{Un} / \mathrm{dl}$ (unidade de fator por decilitro) podendo variar de 50 a $180 \mathrm{Un} / \mathrm{dl}$ circulantes no sangue. Já os níveis de severidade da doença quanto aos tipos de deficiência de fator coagulante, foram considerados segundo Kasper (1996) Pacientes com hemofilia severa ou grave têm em torno de $1 \%$ por Un/dl ou menos e tendem a ter hemorragias espontâneas freqüentes; pacientes com hemofilia moderada têm entre 2 a $4 \%$ de fator por $\mathrm{Un} / \mathrm{dl}$ e apresentam processos hemorrágicos por ocasião de traumas moderados, pacientes com hemofilia leve têm entre 5 a $30 \%$ de fator por Un/dl e apresentam processos hemorrágicos em casos de traumas severos ou em operações cirúrgicas. Contudo, em casos de difícil diagnóstico o nível de severidade foi descrito no prontuário como "indeterminado". As regiões de acometimento de sequielas e deformidades estavam descritas literalmente, e um mesmo paciente poderia possuir uma ou mais alterações em locais diferentes do corpo.

As considerações éticas foram obedecidas através da obtenção de autorização formal junto à coordenação do setor de farmácia e transfusão do serviço de hematologia do HEMOES de acordo com a portaria 196/96 do Ministério da Saúde, pois se trata de pesquisa com dados confidenciais de seres humanos.

O tratamento estatístico dos dados foi realizado no Sistema Operacional Windows XP Professional pelo programa Microsoft Office Excel 2003 que permitiu a realização e a descrição do percentual das variáveis investigadas em gráficos.

\section{RESULTADOS}

Considerando o número de hemofílicos cadastrados no Estado do Espírito Santo, em torno de 400, verifica-se que a amostra de 161 corresponde 40,25\% do total. Após a análise dos dados no setor de farmácia e transfusão do HEMOES, constataram-se os percentuais da hemofilia de acordo com o sexo, tipo de coagulopatia, tipo de deficiência do fator coagulante e de acordo com as áreas de 
acometimento das deformidades e incapacidades mais freqüentes.

A maioria dos pacientes portadores de hemofilia atendidos no HEMOES era do sexo masculino, totalizando 157 prontuários investigados, enquanto apenas 4 prontuários era de pacientes do sexo feminino. Isso já era esperado devido às características genéticas da manifestação da doença.

Quanto ao tipo de coagulopatia, conforme a Gráfico 1, o percentual dos portadores de hemofilia A (deficiência do fator VIII) foi maior em relação ao de hemofilia B (deficiência do fator IX).

Gráfico 1 - Percentual de hemofílicos segundo o tipo de coagulopatia, HEMOES, 2001-2004.

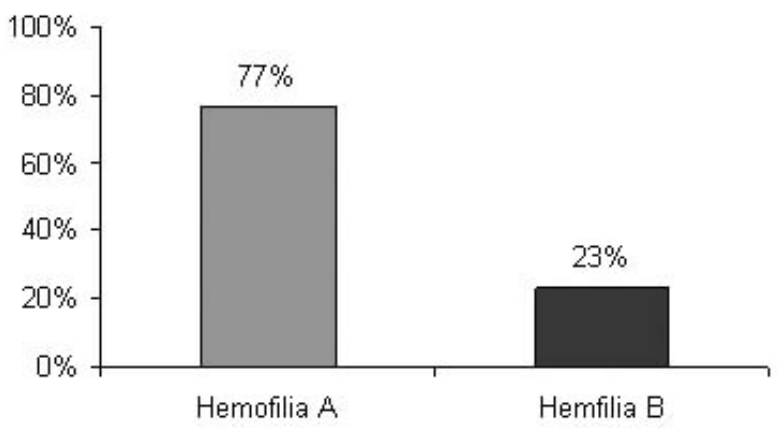

Quanto aos níveis de severidade, verificou-se a predominância dos tipos de deficiência grave e moderada que somados totalizam $43 \%$ dos casos, enquanto o tipo leve representou $16 \%$ dos casos atendidos, conforme a Gráfico 2. Entretanto, deve-se ressaltar que dos 161 prontuários investigados, 63 prontuários (41\%) ainda possuíam indeterminação quanto do tipo de deficiência.

Gráfico 2 - Percentual de hemofilia quanto ao tipo de deficiência do fator, HEMOES, 2001-2005.

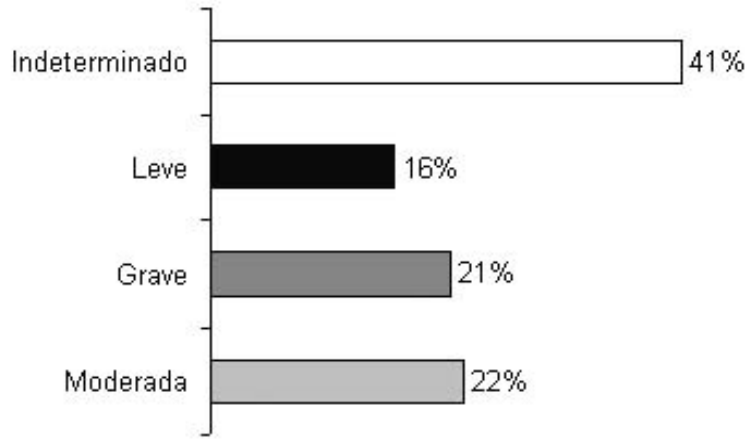

Quanto às áreas de acometimento da hemofilia, totalizaram-se 248 alterações em regiões diferentes. Conforme a Gráfico 3, as áreas mais afetadas foram as articulações do joelho (33\%), cotovelo (17\%), tornozelo $(15,5 \%)$, mãos $(9 \%)$, ombros (5\%), punho (2,5\%) e quadril (2\%) dos casos atendidos, sendo ainda freqüente os acometimentos musculares (11\%) e de mucosas $(5 \%)$.

Gráfico 3 - Acometimento das deformidades e incapacidades nos hemofílicos do HEMOES, 2001-2004.

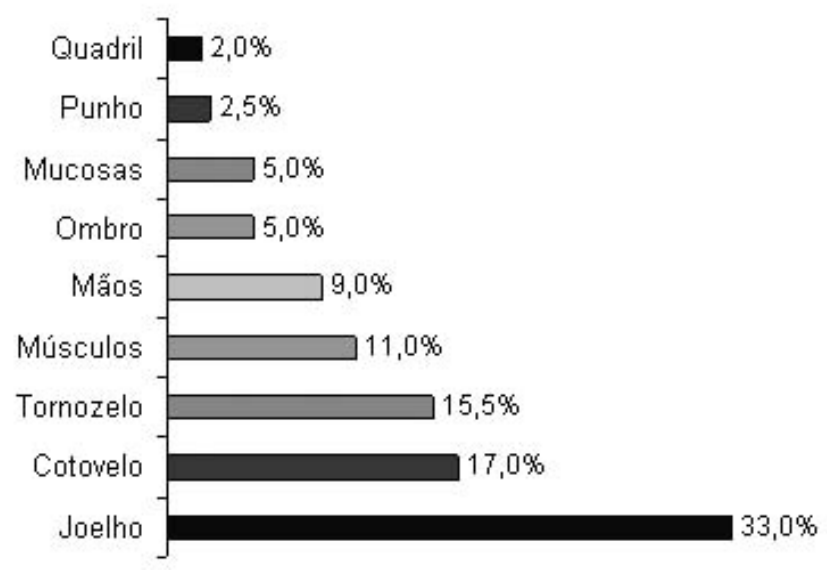

\section{DISCUSSÃO}

Os resultados apresentados mostram que a maioria dos pacientes hemofílicos atendidos no HEMOES possui incapacidades, algumas já irreversíveis, que alteram seu estilo de vida e sua inserção na vida social. Também evidenciaram a necessidade de um atendimento multidisciplinar que ofereça um tratamento específico centrado nas dificuldades e incapacidades mediante seu desempenho e modo de vida diário, restabelecendo suas capacidades e potencialidades para terem melhor qualidade de vida.

Concordando com Lorenzi et al. (1999); Prado et al. (1999); Caio et al. (2001), de que a hemofilia manifesta-se exclusivamente no sexo masculino devido à transmissão hereditária do cromossomo X (recessivo) da mãe portadora aos seus descendentes, os dados comprovaram a essa característica de manifestação genética da doença, pois 98\% do prontuários investigados no HEMOES eram de pacientes do sexo masculino e apenas $2 \%$ do sexo feminino. Já em relação a ocorrência dos tipos de coagulopatias, os achados são semelhantes aos descritos por Lorenzi et al. (2003), quando relata hemofilia A como a mais freqüente prevalecendo em cerca de $85 \%$ dos casos enquanto a hemofilia B em $15 \%$. 
Quanto aos níveis de severidade, Kasper (1997) encontrou a hemofilia do tipo grave ou severa em $50 \%$ dos casos, a hemofilia moderada em $30 \%$ e a hemofilia leve em $20 \%$ dos pacientes estudados. Os resultados apresentados no Gráfico 2 são muito diferentes e revelam um alto índice de indeterminação de classificação (41\%). Isso mostra que algum problema pode estar ocorrendo no serviço de diagnóstico desses pacientes que está impossibilitando a junção dos resultados laboratoriais com as manifestações clínicas da doença para a correta classificação. O diagnóstico preciso e precoce assume sua importância em crianças ou adolescente hemofílicos no que se refere à orientação familiar minimizando erros de conduta que levem a repercussões futuras desfavoráveis como situações de riscos de traumatismos violentos desnecessários e ou extrema superproteção. (Lorenzi et al., 2003)

Nota-se que a demanda de pacientes com incapacidades e deformidades em regiões do corpo é grande (89\% de acometimentos articulares e 11\% musculares) revelando a necessidade atendimento que englobe suas necessidades físicas, psicológicas e sociais. Resultados semelhantes a estes foram encontrados por Matta e Silva (2000) e por Beeton e Buzzard (2000) em seus estudos mostrando que $90 \%$ dos processos hemorrágicos ocorreram no sistema músculo-esquelético, destes $10 \%$ eram musculares e $80 \%$ articulares (hemartroses). Isso prova que o aparelho locomotor é o mais acometimento podendo produzir seqüelas altamente incapacitantes.

Para Falcão et al. (2001) os hematomas musculares constituem a segunda causa mais comum de sangramento em pacientes hemofílicos, sendo mais freqüente na musculatura da panturrilha, coxas, glúteos e antebraço (grupo flexor). Estes, quando afetados, podem resultar em paralisias (nervo mediano e ulnar, no antebraço, nervo fibular, na panturrilha) ou a contraturas isquêmicas (Síndrome de Volkman na mão) e deformidades fixas em equinovaro do tornozelo. As alterações anatômicas acompanhadas de contraturas musculares e, por vezes lesões nervosas associadas ao quadro de instabilidade, alteram definitivamente a biomecânica articular, caracterizando a artropatia hemofílica. (MATTA; SILVA, 2000, p. 1). A artropatia hemofílica crônica reflete o estágio final da hemartrose e é caracterizado por perda da movimentação articular, aparecimento de contraturas fixas em flexão e atrofias musculares, secundárias ao desuso. As articulações mais freqüentemente acometidas envolvem membros inferiores e superiores, sendo as articulações do tornozelo, joelho, quadris, ombro, cotovelo e punho.

Essas incapacidades e deformidades geradas pela doença podem afetar ainda a imagem corporal, a autoconfiança, levar a perda da independência e da segurança na vida pessoal e profissional, a insatisfação com relação ao estilo de vida, a depressão, ao medo e a ansiedade, ao isolamento social e a motivação para o desempenho nas atividades do cotidiano gerado por sentimentos e rotulagens próprias da doença. Por isso as intervenções em terapia ocupacional devem estar baseadas numa relação de parceria, comunicação e cooperação entre o paciente e os profissionais envolvidos, a fim de proporcionar ao paciente à expressão de seus sentimentos e angústias, para que este encontre uma forma de adequação e enfrentamento dos problemas e das limitações (BOSCHEINEN-MORRIN et al., 2002; DE CARLO; LUZO, 2004).

O tratamento da hemofilia, geralmente é realizado nos centros especializados (hemocentros) que possuem um atendimento terapêutico de reposição dos fatores de coagulação, atendendo as intercorrências diárias e serviço ambulatorial de acompanhamento das manifestações clínicas da doença. Entretanto, portador de hemofilia vive em constante necessidade de cuidados específicos e certa dependência quanto à reposição de fatores de coagulação, e isso, causa uma situação psicossocial peculiar para esse paciente que interfere no ambiente familiar e social. Dados da literatura internacional citados por Caio et al. (2001), indicam que os indivíduos com hemofilia costumam ter algumas características psicossociais próprias, embora não específicas, tais como o absenteísmo escolar (WOOLF et al., 1988), retraimento frente aos jogos infantis que envolvem atividades corporais (LOGAN et al., 1993), negação do risco de traumatismo (OREMBAND, 1988), traços masoquistas e relutância à independência pessoal (GUTTON, 1976), problemas sociais e abuso de medicamentos (WEISS et al., 1991), compensação da limitação física pelo desenvolvimento intelectual (HANDFORD et al., 1986), tendência à depressão e à ansiedade (MOLLEMAN; VAN KNIPPENBERG, 1987), personalidade depressiva e pouco dominadora, com maior tendência a comportamentos neuróticos (STEINHAUSEN, 1976). Por outro lado, alguns estudos também demonstram que as características psicossociais dos portadores da hemofilia dependem muito da sua dinâmica familiar (HANDFORD et al., 1986), da possibilidade de conseguirem emprego regular (MARKOVA et al., 1980), da exposição a fatores estressantes do ambiente (BAIRD; WADDEN, 1985) e do atendimento recebido em centro especializado (HERNANDEZ et al., 1989). Esses sentimentos podem levar a repercussões na auto-estima, na adequação social e qualidade de vida do portador de hemofilia.

A hemofilia acarreta em incapacidades na esfera física, 
psicológica e social do indivíduo que vão interferir na autonomia e independência para realizar as atividades do cotidiano (CREPEAU; NEISTALT, 2002). Então a reabilitação, deve ser vista como um processo interdisciplinar e como uma forma de integrar as terapias físicas e psicossociais (MAGALHÃES; PÁDUA, 2003).

Nesse contexto, a reabilitação em Terapia Ocupacional ao portador de hemofilia, baseado na intervenção em ortopedia e traumatologia, segundo De Carlo e Luzo (2004) e Teixeira et al. (2003) visa à prevenção de deformidades, ao treino da independência nas atividades de vida diária e do cotidiano, à confecção de órteses e/ou adaptações e à estimulação das habilidades e potencialidades do indivíduo. A partir dos dados apresentados, é possível identificar que os métodos e recursos terapêuticos utilizados na terapia ocupacional, podem contribuir de forma significativa na vida dos pacientes portadores de hemofilia atendidos nos Centros de Hemoterapia e Hematologia dos diversos estados brasileiros, objetivando: melhorar o desempenho ocupacional/funcional nas atividades de vida diária, de vida prática, atividades de lazer e/ou laborativas e reorganização do cotidiano; estimular a socialização e a auto-estima; garantir a autonomia do indivíduo e sua inserção social; prevenir contraturas e deformidades; "auxiliar o paciente a se adaptar com sucesso ao ambiente, às rupturas em seu estilo de vida e às consequiências da doença, utilizando para isto equipamentos de tecnologia assistiva, como as órteses e as adaptações" (MELVIN, 1989); promover bem estar e melhora na qualidade de vida do indivíduo e de sua família.

Para Lee et al. (2000) uma equipe especializada em reabilitação de hemofílicos deve conter as seguintes especialidades: Fisiatra, Ortopedista, Hematologista, Enfermeira, Fisioterapeuta, Terapeuta Ocupacional, Assistente Social, Psicólogo e Cirurgião-dentista. Esses profissionais devem trabalhar em equipe multidisciplinar com foco no objetivo de atingir o pleno desenvolvimento desses pacientes melhorando sua qualidade de vida. Porque apesar da doença se manifestar exclusivamente nos aspectos físicos, não se podem desconsiderar os demais aspectos desses pacientes.

A partir desse pressuposto, a Terapia Ocupacional junto ao paciente hemofílico poderá atuar através das seguintes intervenções:

\section{1) Estimular as potencialidades e habilidades que o indivíduo possui}

É papel do Terapeuta Ocupacional incentivar o indivíduo a continuar agindo sobre o mundo "mantendo sua capacidade de criação, expressão e produção, de forma que possa conhecer, superar ou minimizar o impacto de suas limitações no cotidiano e fazer surgir e transparecer suas capacidades e potencialidades" (TEIXEIRA et al. 2003, p. 408).

\section{2) Prevenir o aparecimento ou progressão de deformidades e incapacidades}

O tratamento em terapia ocupacional na reabilitação física com pacientes hemofílicos deve incluir procedimentos como a manutenção do posicionamento adequado do membro acometido, o controle do edema, a realização de exercícios de movimentação ativa e passiva, prevenção das deformidades e incapacidades, a manutenção ou aumento da mobilidade articular e força muscular, a confecção de órteses e/ou adaptações e a restituição da função (BARTALOTTI; DE CARLO, 2001; LEE et al., 2000; TEIXEIRA et al., 2003; DE CARLO; LUZO, 2004).

Para controle do edema decorrente das hemorragias, várias técnicas são utilizadas, dentre elas a realização de exercícios ativos de amplitude de movimento articular, elevação do membro e prescrição de atividades em que este tenha de se manter elevado, compressão dos tecidos por meio de massagem retrógrada, enfaixamento compressivo e luvas de compressão e a realização de atividade com objetos gelados ou submersos em água para favorecer a vasoconstricção (BARTALOTTI; DE CARLO, 2001; BOSCHEINEN-MORRIN et al., 2002; LEE et al., 2000; TEIXEIRA et al., 2003; DE CARLO; LUZO, 2004).

\section{3) Intervir sobre aspectos psicossociais}

Um programa de reeducação funcional fornecido ao paciente deve ser traçado no sentido de seu contexto individual, visando ser aceitável e apropriado para suas motivações, interesses, capacidades e perspectivas, no entanto cabe ao terapeuta ocupacional enfocar as capacidades e habilidades do indivíduo propiciando, por meio da atividade e do fazer, a restauração de suas habilidades sociais (CREPEAU; NEISTALT, 2002; DE CARLO;LUZO, 2004).

\section{4) Promover a independência nas atividades de vida diária, de trabalho e de lazer utilizando adaptações, se necessária \\ Cabe ao Terapeuta Ocupacional orientar e planejar junto ao paciente e aos familiares as estratégias de simplificação de tarefas e conservação de energia além de adaptações ambientais e de utensílios específicos (BARTALOTTI; DE CARLO, 2001). É válido ressaltar que na prescrição de uma adaptação o terapeuta ocupacional considere as necessidades do paciente, as questões emocionais, sociais, econômicas e de espaço físico em que}


este se encontra, para que todos (paciente e familiares) "estejam em consonância quanto à adoção dessas modificações e na forma como sistematizá-las e incorporálas à rotina" (TEIXEIRA et al., 2003, p. 415).

Todas essas reflexões possibilitam entender porque se torna essencial a implantação do serviço de terapia ocupacional no HEMOES junto aos portadores de hemofilia para a complementação da equipe multidisciplinar quanto ao atendimento integral em saúde, para restauração das habilidades e potencialidades do portador de hemofilia.

\section{CONCLUSÃO}

O presente trabalho permitiu identificar o perfil dos pacientes hemofílicos atendidos no Centro de Hemoterapia e Hematologia do Espírito Santo, destacando as principais seqüelas e incapacidades que acometem esses pacientes. Somando-se a isso ressalta a necessidade de uma abordagem terapêutica ocupacional como forma de contribuição na reabilitação do portador de hemofilia, utilizando atividades terapêuticas como forma de prevenir deformidades, restabelecer a função, favorecer independência e proporcionar melhora na qualidade de vida. Isso através da estimulação à autonomia no desempenho ocupacional das atividades do cotidiano favorecendo o surgimento das potencialidades e habilidades, já que as incapacidades e deformidades da doença existem e geram repercussões na vida do indivíduo.

Outros estudos também necessitam ser realizados a fim destacar aspectos relevantes acerca da implantação do serviço de terapia ocupacional na assistência ao portador de hemofilia. Com o objetivo de despertar alguns aspectos fundamentais como o apoio da institucional junto ao Ministério da Saúde, envolvimento da equipe multidisciplinar, interesse de investimento, suporte e condições necessárias para a implantação do serviço. Além disso, a preocupação final de ser a de oferecer condições de acesso ao tratamento, assistido pela família, o mais precocemente possível, proporcionando um atendimento mais humanizado com prevenção e limitação dos danos.

SANTOS E. G.; PORTES L. L.; SANTANA A. G.; SANTOS NETO E. T. Deformities and disabilities of hemophilics undergoing treatment at the Hemoterapy and Hematology Centre of Espirito Santo, Brazil. Rev. Ter. Ocup. Univ. São Paulo, v. 18, n. 2, p. 86-94, maio/ago., 2007.

\begin{abstract}
Hemophilia is a severe hereditary disorder that affects blood clotting; it can cause several permanent or transitory deformities and disabilities as well as psychosocial disorders on those affected. The proposition of this is study is not only to identify the main complications found on hemophilic individuals served by Espírito Santo's Hemotherapy and Hematology Centre (HEMOES), but also discuss the necessity of insertion of a Occupational Therapy service as an everyday practice on the integral treatment of those diagnosed with hemophilia. 161 medical records of patients served by HEMOES from 2001 to 2004 were analyzed and complications related to hemophilia were extracted according to a previously defined protocol. The 161 medical records analyzed represent $40 \%$ of the total hemophiliacs registered at Espirito Santo state. Most of them were male (98\%) and the most common type of disorder was TYPE A $(77 \%)$. As for severeness levels, the highly and moderately severe prevailed, adding up to $43 \%$ of the cases, the least severe cases represented $16 \%$. Deformities and disabilities of the articulatory system corresponded to $89 \%$ of the complications while the muscular system had $11 \%$. It can be concluded that the profile of hemophiliac patients taken care by HEMOES is marked by high percents of deformities and disabilities. This situation urges for professional follow up from the biological up to the psychosocial levels and highlights the importance of insertion of the occupational therapist on the multiprofessional staff that cares for hemophiliacs, so that they can be treated integrally.
\end{abstract}

KEYWORDS: Hemophilia A/complications. Hemotherapy service. Complications. Occupational therapy.

\title{
REFERÊNCIAS
}

Agencia de Vigilância Sanitária - ANVISA. Relatório Estatístico do Cadastro de Coagulopatias Hereditárias. Gerência-geral de Sangue, outros Tecidos e Órgãos. Brasília: Ministério da Saúde, 2002. Disponível em: <http://www.anvisa.gov.br/sangue/p- hemofilia/programa.pdf>. Acesso em: 24 abr. 2004.

BAIRD, P.; WADDEN, T. A. Effects of life stressors on blood usage in hemophiliac patients: a pilot study. Psychosomatics, 1985. 
BARTALOTTI, C.C.; DE CARLO, M.M. R. P. (Org.). Terapia ocupacional no Brasil - fundamentos e perspectivas. São Paulo: Plexus, 2001.

BEETON, K.; BUZZARD, B. Physiotherapy management of haemophilia. USA: Blackwell Science, 2000.

BONFIM, M.; CAMPOS, K.C. O processo de doação de sangue: a experiência de captação no HEMOES, 2001. Trabalho de Conclusão de Curso (Graduação em Serviço Social) - Centro de Ciências Jurídicas e Econômicas, Departamento de Serviço Social, Universidade Federal do Espírito Santo, Vitória, 2001.

BRASIL. Ministério da Saúde. Agencia Nacional de Vigilância Sanitária. Manual técnico para investigação da transmissão de doenças pelo sangue. Brasília: Ministério da Saúde, 2003.

BRASIL. Resolução da Diretoria Colegiada (RDC) $\mathrm{n}^{\circ}$ 153, de 14 de junho de 2004. Dispõe sobre as normas técnicas da hemoterapia. Ministério da Saúde. Secretaria de Assistência à saúde. Departamento de assistência e promoção à saúde Coordenação de Sangue e Hemoderivados. Coletânea das legislações pertinentes às atividades hemoterápicas e hematológicas. Diário Oficial [da] República Federativa do Brasil, $\mathrm{n}^{\circ} 049,18$ jun. 1997.

BRASIL. Portaria interministerial $n^{\circ} 07 / 80$ de 30 de abril de 1980. Dispõe sobre a criação do programa de sangue e hemoderivados - Pró-sangue. Ministério da Saúde. Secretaria de Assistência à saúde. Departamento de assistência e promoção à saúde Coordenação de Sangue e Hemoderivados. Coletânea das legislações pertinentes às atividades hemoterápicas e hematológicas. Diário Oficial [da] República Federativa do Brasil, no 049,18 jun. 1997.

BOSCHEINEN-MORRYN, J.; CONOLLY, W. B.; DAVEY, V. A mão bases da terapia. 2a. ed. São Paulo: Manole, 2002.

CAIO, V.M. et al. Genética comunitária e hemofilia em uma população brasileira. Caderno de Saúde Pública, Rio de Janeiro, v. 17, n. 3, mai. /jun. 2001.

CREPEAU, E.B.; NEISTALT, M. E. Terapia ocupacional. Rio de Janeiro: Guanabara Koogan, 2002.

DE CARLO, M.M. R. P.; LUZO, M.C. M. Terapia Ocupacional - reabilitação física e contextos hospitalares. São Paulo: Roca, 2004.

FALCÃO, R.P.; PASQUINI, R.; ZAGO, M.A. Hematologia: fundamentos e prática. São Paulo: Atheneu, 2001.

GUTTON, P. Reflections on the psychology of the hemophiliac children. Rev. Hyg. Mental. l'Enfance, 1976.
HANDFORD, H. A.; MAYES, S. D.; BAGNATO, S. J.; BIXLER, R. O.; MATTISON, R. E. Relationships between variations in parent's attitudes and personality traits of hemophilic boys. Am. J. Orthopsychiatr., v. 56, n. 3, p. 424434, 1986.

HEMOES. Secretaria de Estado da Saúde. Centro de Hemoterapia e Hematologia do Espírito Santo. Planejamento estratégico HEMOES. Vitória: Instituto de Saúde Pública, 2001.

HERNANDEZ, J.; GRAY, D.; LINEBERGER, H. P. Social and economic of wellbeing among hemophiliacs over a 5 years period. Gen. Hosp. Psychiatr., 1989.

KASPER, C. Desordens hereditárias do fator de coagulação sanguíneo e o seu tratamento. São Paulo: Federação Brasileira de Hemofilia, 1997. Disponível em: 〈http:// www. wfh.org〉. Acesso em: 12 abr. 2004.

LEE, C. A.; GODDARD, N. J.; RODRIGUEZ-MERCHAN, E.C. Musculoskeletal aspects of haemoplilia. USA: Blackwell Science, 2000.

LOGAN, F. A.; GIBSON, B.; HANN, I. M.; PARRY-JONES, W. L. Children with hemophilia: same or different? Child-Care, Health and Development, 1993.

LORENZI, T. F. et al. Manual de hematologia: propedêutica e clínica. 3a. ed. São Paulo: Medsi, 2003.

LORENZI, T. F.; NETO, S. W. VERASTRO, T. Hematologia e hemoterapia: fundamentos de morfologia fisiologia, patologia e clínica. São Paulo: Atheneu, 1999.

MAGAlHAES, L.V.; PÁdUA, E.M. M. (Org.). Terapia ocupacional teoria e prática. São Paulo: Papirus, 2003.

MARKOVA, I.; LOCKYER, R.; FORBES, C. D. Self-perception of employed and unemployed hemophiliacs. Psychol. Med., 1980.

MATTA, M.A.P.; SILVA, J.B.J. A fisioterapia no contexto de tratamento do paciente hemofílico. Disponível em: <http:// www.hemocam.hpg.ig.com.br/nfisio.htm>. Acesso em: 12 Ago 2004.

MELVIN, J. L. Roles and functions of occupational therapy in the management of patients with rheumatic diseases. Rheumatic Diesease in the adult and child: occupational therapy and rehabilitation. 3a. ed. Philadelphia: F. A. Davis, 1989.

MOLLEMAN, E.; VAN KNIPPENBERG, A. Social and psychological aspects of haemophilia. Patient Education and Counseling, 1987 apud CAIO, Vânia M. et al. Genética comunitária e hemofilia em uma população brasileira. Cad. Saúde Pública, Rio de Janeiro, v. 17, n. 3, mai. /jun. 2001. 
SANTOS, E. G. et al. Deformidades e incapacidades dos hemofílicos. Rev. Ter. Ocup. Univ. São Paulo, v. 18, n. 2, p. 86-94, maio/ago., 2007.

OREMBAND, E. K. Work dynamics in family care of hemophilic children. Soc. Med., v. 26, n. 4, p. 467-475, 1988.

PRADO, F.C.; RAMOS, J.A.; VALLE, J.R. Atualização terapêutica: manual prático de diagnóstico e tratamento. 19a. ed. São Paulo: Artes Médicas, 1999.

STEINHAUSEN, H. C. Hemophilia: a psychological study in chronic disease in juveniles. J. Psychosom. Res., v. 20, n. 5, p. 461-467, 1976.

TEIXEIRA, E. et al. Terapia ocupacional na reabilitação física. São Paulo: Roca, 2003.

WEISS, H. M.; SIMON, R.; LEVI, J.; FORSTER, A.
Compliance in a comprehensive hemophilia center and its implication for home care. Family Systems Medicine, 1991.

WILLIAM, W.J. et al. Hematology. USA: International Edition, 1990.

WINTROBE. Hematologia clínica. v. 2. Manole: São Paulo, 1998.

WOOLF, A.; RAPPAPORT, L.; REARDON, P.; CIBOROWSKI, J. School functioning and disease severity in boys with hemophilia. J. Dev. Behav. Pediatri., 1988.

World Federation of Haemoplilia. Passaporte global treatment centre directory. United States of América: Passaporte, 2002.

Recebido para publicação: Mar./2007

Aceito para publicação: Abr./2007 